\title{
Alginate Biopolymer Coatings Obtained by Electrophoretic Deposition on Ti15Mo Alloy
}

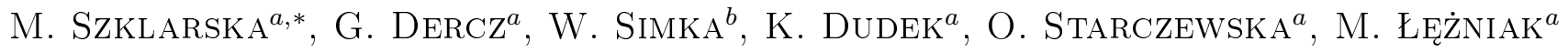 \\ AND B. ŁOSIEWICZ ${ }^{a, *}$
}

${ }^{a}$ Institute of Materials Science, Silesian Interdisciplinary Centre for Education and Research University of Silesia, 75 Pułku Piechoty 1A, 41-500 Chorzów, Poland

${ }^{b}$ Faculty of Chemistry, Silesian University of Technology, B. Krzywoustego 6, 44-100 Gliwice, Poland

\begin{abstract}
In this work, the electrophoretic deposition method has been developed for the fabrication of bioactive alginate coatings on the surface of Ti15Mo implant alloy. Thin $\mathrm{ZnO}$ film was deposited cataphoretically as the interlayer prior to anaphoretic deposition of alginate (Alg) which was performed from aqueous solution containing $1 \mathrm{~g} \mathrm{dm}^{-3}$ of NaAlg at room temperature. The deposition voltage and time varied in the range $20-50 \mathrm{~V}$ and $30-120 \mathrm{~min}$, respectively. The microstructure of Alg coatings was studied by scanning electron microscope, and the surface roughness was analysed using atomic force microscopy. Structure was studied by grazing incidence X-ray diffraction. Chemical composition and functional group were examined using energy dispersive spectrometry and attenuated total reflectance Fourier transform infrared spectroscopy methods, respectively. It was found that controlling the deposition conditions it is possible to obtain amorphous Alg coatings of variable thickness and porosity. Mechanism of electrophoretic deposition of bioactive Alg coatings on the Ti15Mo alloy surface was discussed.
\end{abstract}

DOI: 10.12693/APhysPolA.125.919

PACS: 87.18.Fx, 87.85.J-, 82.35.Pq, 87.15.rp, 81.15.-z

\section{Introduction}

Titanium and Ni-free Ti-based alloys, are now one of the most studied and sought-metallic materials for use in medicine due to their unique properties like high corrosion resistance, biological indifference, low specific gravity and excellent mechanical properties (low Young's modulus, high fatigue strength) [1]. These features make the biomaterials based on such titanium alloys attractive materials for the production of implants both short- and long-term such as stents, wire orthodontics, dental implants, knee prosthesis, hip, plates, bone screws, etc. [2].

Recently, TiMo alloys have gained huge popularity for medical applications. Addition of molybdenum is an excellent stabilizer of $\beta$-titanium (body-centred cubic crystal structure) phase. Furthermore, Mo reduces the modulus of elasticity and increases corrosion resistance as well as biocompatibility due to the ability to spontaneous passivation resulting in oxide layer formation which is characterized by a high electrochemical stability [3, 4]. This layer has the ability to self-repair if damaged, known as self-passivation. Thanks to these features TiMo alloys are more suitable for biomedical applications than conventional biomaterials [1].

Nowadays, to enhance biocompatibility of the metallic implant, the surface modification by deposition of bioactive polymer coatings has been proposed in literature [5]. Recent studies have shown significant interest

\footnotetext{
*corresponding author; e-mail: mszklarska@us.edu.pl, bozena.losiewicz@us.edu.pl
}

in electrophoretic deposition (EPD) of polysaccharides such as alginic acid $(\mathrm{Alg} \mathrm{H})$ also called algin or alginate (Alg) on conductive substrates as 316L stainless steel, gold and even on semi-conductive surface of titanium or NiTi shape memory alloy [5-7].

$\mathrm{Alg}$ is natural biodegradable polymer characterized by high biocompatibility, bio-functionality, non-toxicity and low-cost. It is widely used in medical applications such as tissue engineering, drug delivery, nervous tissue repair, bone or wound dressing $[7,8]$. Alg as anionic polymer with carboxyl end groups is also attractive biomaterial for the production of composite ceramic-polymer coatings by EPD.

The great advantage of EPD is that this method does not need high-tech equipment, infrastructure and it is cost-effective [5]. EPD has recently gained increasing interest both in academia and industrial sector for novel applications in the processing of advanced bioactive coatings.

However, there is still a lack of literature data concerning EPD of Alg coatings on the surface of TiMo implant alloy. Our attempts to obtain $\mathrm{Alg}$ coating directly on the surface of Ti15Mo alloy by anaphoretic deposition did not give satisfactory results due to the presence of oxide film formed at the anode surface. Resistivity of titania at $25^{\circ} \mathrm{C}$ is $10^{13} \Omega \mathrm{cm} \mathrm{[9]}$. Therefore, we have proposed in this work prior to Alg deposition, to deposit cataphoretically a thin film of zinc oxide as an interlayer. Resistivity of thin $\mathrm{ZnO}$ film strongly depends on the production method and it has been reported to be of order of $1500 \Omega \mathrm{cm}$ to $10^{9} \Omega \mathrm{cm}$ [10]. Zinc is a good antibacterial agent, its presence stimulates bone growth, promotes osseointegration, and its deficiency is associated with de- 
velopment of osteoporosis [11, 12]. Also high isoelectric point of zinc oxide $(\mathrm{pH}=9.5)$, which is responsible for the electrostatic immobilization of enzymes at physiological $\mathrm{pH}$, has made it attractive for biomedical applications [13].

The main purpose of this work was to obtain anaphoretically deposited Alg coatings on the surface of Ti15Mo alloy coated with thin $\mathrm{ZnO}$ interlayer, and physical and chemical characterization of the Alg deposits in dependence on the EPD parameters.

\section{Experimental}

Sodium alginate $(\mathrm{AlgNa})$ and zinc acetate $\left(\mathrm{C}_{4} \mathrm{H}_{6} \mathrm{O}_{4} \mathrm{Zn}\right.$, 99\% purity) were purchased from Acros Organics. Nanopure water (Milli-Q, $18.2 \mathrm{M} \Omega \mathrm{cm}^{2}$ ) was used for the solution preparation. Before EPD, each bath was stirred magnetically for $24 \mathrm{~h}$. Tested samples of Ti15Mo alloy in the form of disks were cut from the rod of $10 \mathrm{~mm}$ in diameter, and grounded with 80 to $5000 \#$ grit silicon carbide paper. The samples were ultrasonically cleaned using acetone and depassivated for $1 \mathrm{~min}$ in solution containing $\mathrm{H}_{2} \mathrm{SO}_{4}\left(4 \mathrm{~mol} \mathrm{dm}^{-3}\right)$ and $\mathrm{HF}\left(1 \mathrm{~mol} \mathrm{dm}^{-3}\right)$ [3], and then rinsed with nanopure water. The sample under investigation was placed inside a self-designed teflon holder which contained O-ring. The exposed surface area of the electrode was $0.64 \mathrm{~cm}^{2}$. EPD process was carried out using a MAG-5N galvanizing aggregate in two-electrode electrochemical cell with the working electrode and a platinum foil as a counter electrode. The distance between electrodes was $15 \mathrm{~mm}$.

Cataphoretic deposition of thin $\mathrm{ZnO}$ film on Ti15Mo electrode was carried out from $5 \mathrm{mM}$ zinc acetate aqueous solution at a constant voltage of $E=20 \mathrm{~V}$ for 5 min at room temperature. Next, the electrode was sonicated in nanopure water for $5 \mathrm{~min}$. On such a prepared substrate, alginate coatings were deposited by anaphoresis from an aqueous solution of $0.1 \%$ sodium alginate. The EPD was performed at a constant voltage of $E=20-50 \mathrm{~V}$ for $t=$ 30-120 min at room temperature. The deposited Alg coatings were dried in air for $48 \mathrm{~h}$ and weighted.

Functional groups of the Alg coatings deposited on Ti15Mo alloy were determined using attenuated total reflectance Fourier transform infrared (ATR-FTIR) spectroscopy (Schimazu IR Prestige-21). Microstructure of the biopolymer coatings was investigated using a JEOL JSM-6480 scanning electron microscope (SEM). Chemical composition was performed by energy dispersive spectrometry (EDS) method. The Hysitron atomic force microscopy (AFM) and Mitutoyo SURFTEST SJ-500 profilograph were used to determine the surface roughness.

The X-ray diffraction (XRD) measurements were performed on the X'Pert Philips PW 3040/60 diffractometer operating at $30 \mathrm{~mA}$ and $40 \mathrm{kV}$, which was equipped with a vertical goniometer and an Eulerian cradle. The wavelength of radiation $\left(\lambda \mathrm{Cu} K_{\alpha}\right)$ was $1.54178 \AA$. The grazing incidence X-ray diffraction (GIXD) patterns were registered in the $2 \theta$ range from $10^{\circ}$ to $50^{\circ}$ with a $0.05^{\circ}$ step for the incident $0.25^{\circ}, 0.50^{\circ}, 1.00^{\circ}$ angle.

\section{Results and discussion}

The deposition mechanism of Alg coatings in the form of gel on the Ti15Mo alloy can be explained by electrophoresis of $\mathrm{Alg}^{-}$macromolecules and their charge neutralization in the region of low $\mathrm{pH}(\mathrm{pH}<3)$ at the anode surface similarly as it was reported by Cheong and Zhitomirsky for alginic acid gel deposition on $\mathrm{NiTi}(50 \% \mathrm{Ni})$ and platinised silicon wafers $[7,8]$.

At first, sodium alginate dissociates in aqueous solution of alkaline $\mathrm{pH}$ forming anionic $\mathrm{Alg}^{-}$species

$\mathrm{NaAlg} \rightarrow \mathrm{Na}^{+}+\mathrm{Alg}^{-}$.

At the anode surface the electrolytic decomposition of water particle takes place and the decrease of $\mathrm{pH}$ could be observed

$$
2 \mathrm{H}_{2} \mathrm{O} \rightarrow \mathrm{O}_{2}+4 \mathrm{H}^{+}+4 \mathrm{e}^{-} \text {. }
$$

Alg coating deposition occurs as a result of electrophoretic motion of $\mathrm{Alg}^{-}$anions toward the anode (Fig. 1), where due to the low $\mathrm{pH}, \mathrm{Alg}^{-}$acromolecules are neutralized forming alginic acid gel [5]:

$$
\mathrm{Alg}^{-}+\mathrm{H}^{+} \rightarrow \text { HAlg. }
$$

HAlg is an anionic polysaccharide. Alginic acid is a linear copolymer with homopolymeric blocks of (1-4)-linked $\beta$-D-mannuronate (M) and its $\mathrm{C}-5$ epimer $\alpha$-L-glucuronate $(\mathrm{G})$ residues, respectively, covalently linked together in different sequences or blocks (Fig. 2).

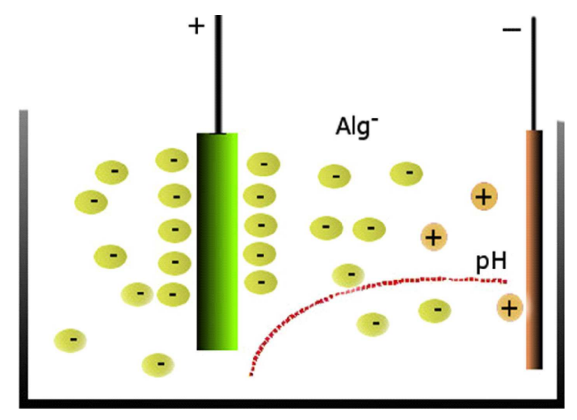

Fig. 1. Scheme of EPD of Alg coating by anaphoresis technique.

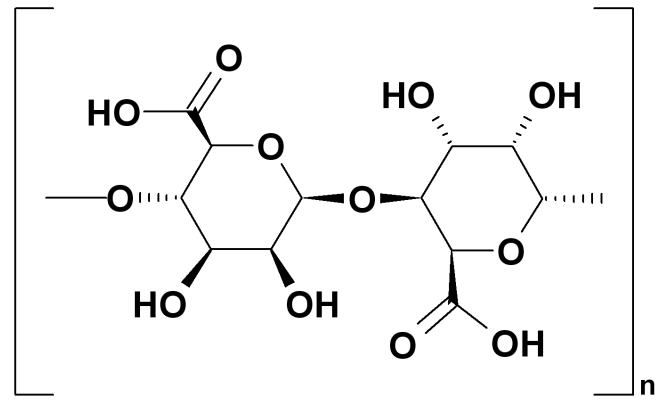

Fig. 2. Structure of alginic acid.

Sodium alginate reagent and the deposited Alg coatings on the Ti15Mo alloy surface with $\mathrm{ZnO}$ interlayer, 
were studied by ATR-FTIR (Fig. 3). The band at $1730 \mathrm{~cm}^{-1}$ is attributed to the stretching of the protonated carboxylic group of alginate [7]. The peaks at $3450 \mathrm{~cm}^{-1}$ and around $1030 \mathrm{~cm}^{-1}$ are characteristic for $\mathrm{OH}$ and $\mathrm{C}-\mathrm{O}-\mathrm{C}$ stretching, respectively. Peaks observed at $1600 \mathrm{~cm}^{-1}$ and $1410 \mathrm{~cm}^{-1}$ correspond to salified carboxylic groups $\left(\mathrm{COO}^{-}\right)$. $\mathrm{C}-\mathrm{O}$ stretching vibrations is $1298 \mathrm{~cm}^{-1}$.

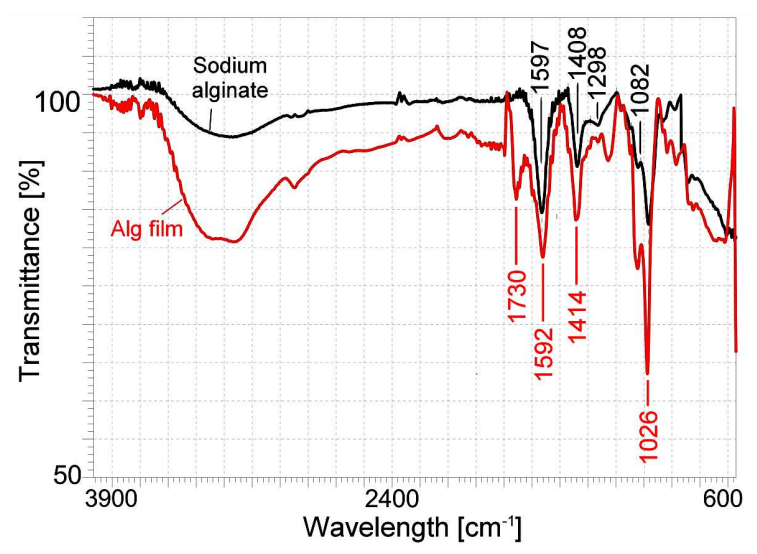

Fig. 3. FTIR spectrum of sodium alignate reagent (black line) and Alg coating deposited at a voltage of $40 \mathrm{~V}$ for $60 \mathrm{~min}$ on the Ti15Mo alloy (red line).

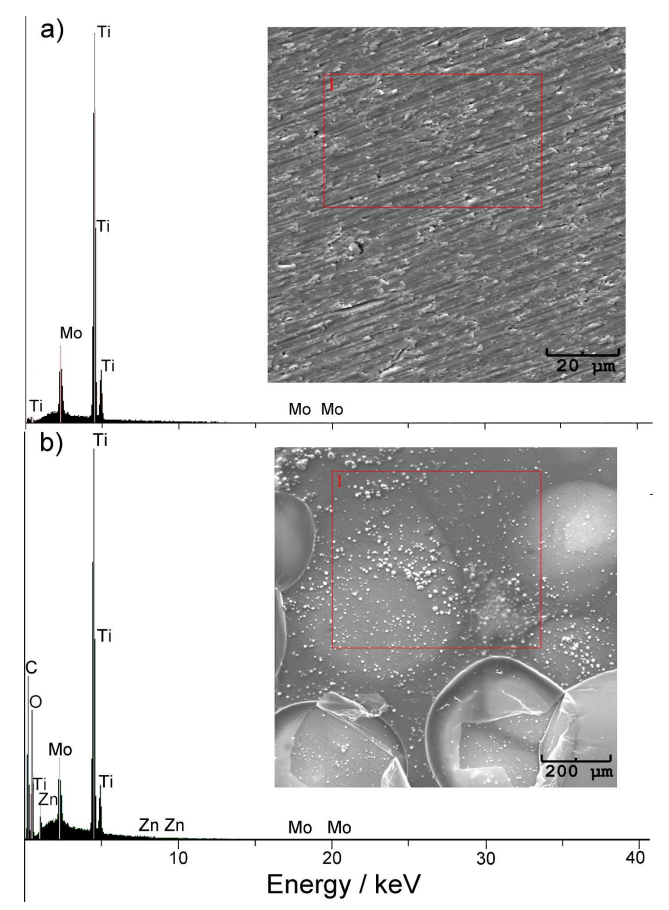

Fig. 4. EDS analysis of the Ti15Mo alloy (a) and the Alg coating deposited at a voltage of $50 \mathrm{~V}$ for 60 min on the Ti15Mo alloy (b).

EDS analysis (Fig. 4) revealed the presence of oxygen and carbon on the Ti15Mo substrate. Based on these results we can confirm the presence of the Alg coating. Also peaks originating from the substrate, such as Ti and Mo, could be observed. These results indicate that the thin coating of Alg was obtained.

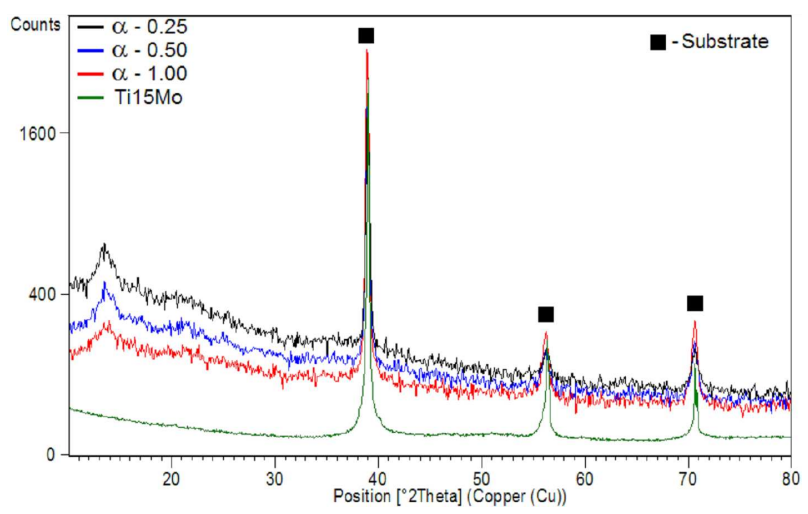

Fig. 5. X-ray diffraction pattern in GIXD geometry at $\alpha=0.25^{\circ}, 0.50^{\circ}, 1.00^{\circ}$ for the Alg coating deposited at a voltage of $50 \mathrm{~V}$ for $60 \mathrm{~min}$ and for the Ti15Mo alloy.
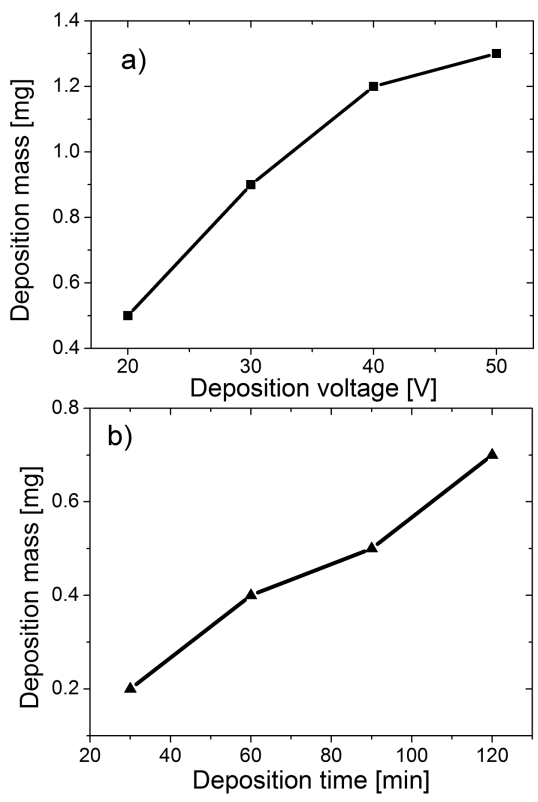

Fig. 6. Alg deposit mass as a function of: (a) deposition voltage at constant time $(60 \mathrm{~min})$, and $(\mathrm{b})$ deposition time at constant voltage $(20 \mathrm{~V})$, from $0.1 \%$ aq sodium alginate solution.

Figure 5 shows an example of XRD pattern of the deposited Alg coating. The broad peak was observed at $2 \theta$ value around $14^{\circ}$, which is characteristic for alginate and indicates the amorphous nature of the Alg deposit. Additional diffraction peaks are corresponding to the substrate of the Ti15Mo alloy. Comparing the received spectrum with the literature data, the presence of alginate coating on the Ti15Mo surface was confirmed [7, 14].

EPD was carried out under different conditions, various deposition time and voltage. All these factors affect 
the thickness of the obtained Alg coatings. In Fig. 6 one can observe that deposit mass increases with increasing time (Fig. 6a) and voltage (Fig. 6b) of electrophoresis.

Roughness studies of the Alg anodic deposits indicate that its degree is affected alike by the time and voltage of electrophoresis. The results obtained using the profilograph over relatively large macroscopic area $2 \mathrm{~mm}$ length revealed that surface roughness increases with increasing voltage of deposition. Roughness index, $R_{\mathrm{a}}$, determined for the Alg coating deposited at a voltage of $50 \mathrm{~V}$ for $60 \mathrm{~min}$ and at a voltage of $20 \mathrm{~V}$ for $120 \mathrm{~min}$, were 6.912 and $0.075 \mu \mathrm{m}$, respectively.
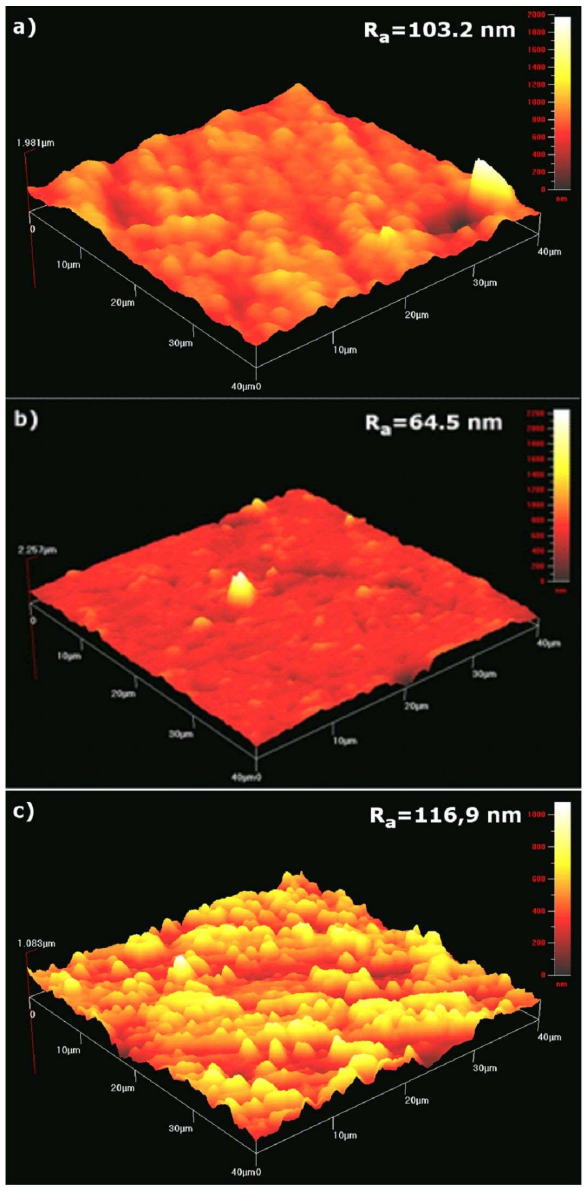

Fig. 7. AFM images of the Alg coating deposited at a deposition voltage of: (a) $50 \mathrm{~V}$ for $60 \mathrm{~min}$, (b) $20 \mathrm{~V}$ for $120 \mathrm{~min}$, and (c) $20 \mathrm{~V}$ for $60 \mathrm{~min}$, on the Ti15Mo alloy.

AFM measurements obtained from the small microscopic area $4 \times 4 \mu \mathrm{m}^{2}$, confirmed the results obtained by the profilograph. $R_{\mathrm{a}}$ index calculated for Alg coatings deposited at a voltage of $50 \mathrm{~V}$ for $60 \mathrm{~min}$ and at a voltage of $20 \mathrm{~V}$ for 120 min were 103.2 and $64.5 \mathrm{~nm}$, respectively (Fig. $7 \mathrm{a}, \mathrm{b}$ ). This method also revealed that the longer deposition time at the same voltage of electrophoresis, leads to decrease of roughness index. It is connected with the thickness of the Alg coating. During the EPD, alginate uniformly coats the substrate sur- face. Therefore, examining the thin film deposited for 60 min, higher roughness of the surface can be observed $\left(R_{\mathrm{a}}=116.9 \mathrm{~nm}\right)$. Along with the deposition time, alginate fills substrate irregularities, resulting in a smoother surface. $R_{\mathrm{a}}$ index calculated for Alg coatings deposited for $120 \mathrm{~min}$ was $64.5 \mathrm{~nm}$ (Fig. $7 \mathrm{~b}, \mathrm{c})$.

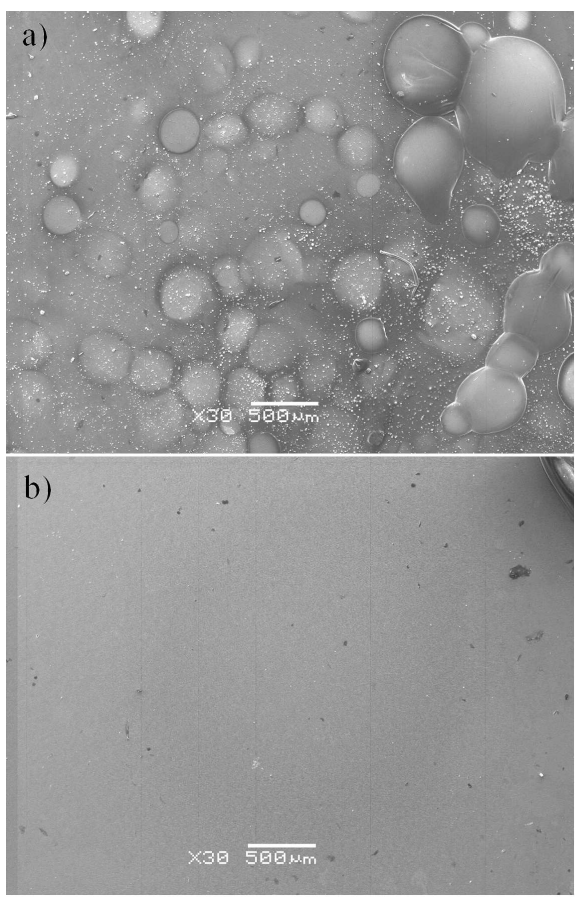

Fig. 8. SEM images of the surface of the Alg coating deposited at: (a) voltage of $50 \mathrm{~V}$ for $60 \mathrm{~min}$, and (b) voltage of $20 \mathrm{~V}$ for $60 \mathrm{~min}$, on the Ti15Mo alloy.

Results obtained by SEM indicate that the value of the voltage used for EPD significantly influences the microstructure of the obtained Alg coatings. Porous coatings were received at high deposition voltage of $50 \mathrm{~V}$ (Fig. 8a). It was formed as a result of a rapid evolution of oxygen at the anode (2). Reducing the voltage, the rate of oxygen evolution decreases, thereby deposited Alg coatings are more uniform, Fig. 8b.

\section{Conclusions}

FTIR, EDS, SEM, AFM, and XRD studies confirmed the possibility of anaphoretic deposition of amorphous alginate coatings on Ti15Mo implant alloy under condition that thin $\mathrm{ZnO}$ film is applied as the interlayer. It was found that the Alg coating microstructure, thickness and defectiveness can be controlled by time and voltage used for the EPD process. The studied EPD of Alg biopolymer in aqueous solution at room temperature is applicable for the surface modification of the Ti15Mo implant alloy to develop novel bioactive coatings.

\section{References}

[1] P. Ducheyne, Comprehensive Biomaterials, Eds.: K.E. Healy, D.W. Grainger, D.W. Hutmacher, C.J. Kirkpatrick, Elsevier Sc., Amsterdam 2011. 
[2] J.R. Davies, Handbook of Materials for Medical Devices, ASM International, 2003.

[3] W. Simka, A. Krząkała, D.M. Korotin, I.S. Zhidkov, E.Z. Kurmaev, S.O. Cholakh, K. Kuna, G. Dercz, J. Michalska, K. Suchanek, T. Gorewoda, Electrochim. Acta 96, 180 (2013).

[4] C.G. Nava-Dino, R.G. Bautista-Margulis, M.A. NeriFlores, M.V. Orozco-Carmona, S.D. de la Torre, J.G. Gonzalez-Rodriguez, J.G. Chacon-Nava, A. Martinez-Villafane, Int. J. Electrochem. Sci. 7 , 4250 (2012).

[5] A.R. Boccaccini, S. Keim, R. Ma, Y. Li, I. Zhitomirsky, J. R. Soc. Interface 7, 581 (2010).

[6] R. Ma, X. Pang, I. Zhitomirsky, Surf. Eng. 27, 693 (2011).

[7] M. Cheong, I. Zhitomirsky, Coll. Surf. A 328, 73 (2008).

[8] D. Zhitomirsky, J.A. Roether, A.R. Boccaccini, I. Zhitomirsky, J. Mater. Process. Tech. 209, 1853 (2009).
[9] R.G. Breckenridge, W.R. Hosler, Phys. Rev. 91, 793 (1953).

[10] K. Ellmer, J. Phys. D, Appl. Phys. 34, 3097 (2001).

[11] P. Petrini, C.R. Arciola, I. Pezzali, S. Bozzini, L. Montanaro, M.C. Tanzi, P. Speziale, L. Visai, Int. J. Artif. Organs 29, 434 (2006).

[12] H. Zreiqat, S.M. Valenzuela, B.B. Nissan, R. Roest, C. Knabe, R.J. Radlanski, H. Renz, P.J. Evans, Biomaterials 26, 7579 (2005).

[13] Y. Li, K. Wu, I. Zhitomirsky, Coll. Surf. A 356, 63 (2010).

[14] J.B. Xu, D.A. Spittler, J.P. Bartley, R.A. Johnson, J. Membrane Sci. 260, 19 (2005). 